Given the increasing success of campaigns to ensure the voice of people with dementia is represented, there are a growing number of people in the early stages of dementia being called upon to account for their experience, as a means of developing a collective illness identity. This paper draws on qualitative interviews with IDEAL pilot study participants who were members of a patient advocacy group and who represent the voices of patients and carers in research. We explore two connected themes: firstly, the ways in which people with dementia participate in identity construction, performing biographical reconstruction to make sense of their lives and preserve their sense of self; and secondly, as representatives of the patient voice, these interviews produce dementia illness narratives that are a source of material and symbolic value. The paper reflects on the dualities and tensions within these different narratives.

\section{LETTING GO OF COHERENCE: THE CHALLENGES OF REPRESENTING DEMENTIA}

A. Hillman ${ }^{2}$, I.R. Jones ${ }^{2}$, C. Quinn ${ }^{1}$, L. Clare ${ }^{1}$, 1. University of Exeter, Exeter, United Kingdom, 2. Cardiff University, Cardiff, United Kingdom

Drawing on IDEAL study in-depth interviews, this paper explores a sociological response to the 'turn to personhood' in qualitative research with people with dementia. Recognizing the voice of the person with dementia has led to important methodological developments to mitigate the practical difficulties of doing research with people for whom recalling events and reflecting on their meaning poses a challenge. This paper suggests that methods, including the qualitative interview, are imbued with a politics of selfhood in which individuals give coherence to experience and emotion. Such a politics jars with representations of dementia as a gradual decline in capacity, including a capacity to speak. The problem of representation in dementia research requires us to re-think method and methodology: firstly, to re-asses expectations of the research encounter; and secondly, to develop alternative interpretations of meaning which support difference in social and temporal frames, rather than seeking to eliminate them.

\section{HOW LONELY AND ISOLATED ARE OLDER PEOPLE WITH DEMENTIA AND THEIR CARERS?}

C. Victor ${ }^{1}$, Y. Wu 2 , R. Jones 3 , M. Kopelman ${ }^{4}$, N. Savitch ${ }^{5}$, J. Pickett ${ }^{6}, \mathrm{~S}$. Nelis ${ }^{2}, 1$. Brunel University, Uxbridge, United Kingdom, 2. University of Exeter, Exeter, United Kingdom, 3. RICE, Bath, United Kingdom, 4. Kings College London, London, United Kingdom, 5. Innovations in Dementia, London, United Kingdom, 6. Alzheimer's Society, London, United Kingdom

Loneliness and/or isolation are key indicators of quality of life and living well. Using data from the IDEAL study we investigated the prevalence of loneliness and isolation for people with dementia and their carers. Loneliness was measured using the six-item de Jong Gierveld scale and isolation by the six-item Lubben social network measure. Preliminary data analysis reports a prevalence of loneliness for people with dementia of $7.8 \%$, comparable with the $10 \%$ reported for the general population of older people aged $65+$, and a prevalence of $31.8 \%$ for carers. Mean network sizes were 15 for people with dementia and 17 for carers, which are comparable with the general population. People with dementia demonstrate levels of social engagement broadly comparable with rest of the population. Carers, whilst reporting network sizes similar to the general population, report very much higher levels of loneliness, highlighting the importance of relationship quality for living well.

\section{SESSION 5040 (PAPER)}

\section{VOLUNTEERING/CIVIC ENGAGEMENT/SOCIAL INCLUSION}

\section{FACTORS ASSOCIATED WITH SOCIAL ACTIVITY PARTICIPATION AMONG MIDDLE-AGED AND OLDER CHINESE IN CHINA}

A. Restorick Roberts ${ }^{1,2}$, X. Pan ${ }^{3}$, Y. Lee ${ }^{4}$, 1. Family Science o Social Work, Miami University, Oxford, Ohio, 2. Scripps Gerontology Center, Oxford, Ohio, 3. Texas State University, San Marcos, Texas, 4. University of Calgary, Calgary, Alberta, Canada

Research from Western samples has linked participation in social, leisure, and other productive activities in later life with better physical and mental health. In China, social activity participation is an understudied, yet important topic. To measure and investigate social activity participation in China, ten items were included in the first wave (2011) of the nationally representative Chinese Health and Retirement Longitudinal Study (CHARLS). In this study, we aimed to (1) classify the dimensions of social activity among middleaged and older Chinese adults through an exploratory factor analysis (EFA), and (2) identify the socio-demographic characteristics associated with each factor through a confirmatory factor analysis (CFA). Results from the EFA identified four dimensions of social activity, including informal volunteering (helping others without organizational affiliation), leisure (interacting with friends), formal volunteering and learning (charity work through an organization and lifelong learning), and technology use. The indices suggested that the hypothesized model fit the data adequately $(1, n=16,224)$ $=13.50, p=.26$; RMSEA=.01; CFI=.99; TLI=.99; $\mathrm{SRMR}=.02$. CFA findings showed that socio-demographic factors including age, education, marital status, and residential region were significantly associated with the informal volunteering factor, but not the other three factors. Respondents who were older $(\beta=.06, p<.01)$, higher educated $(\beta=.04, p<.01)$, married $(\beta$ $=.14, p<.001)$, and living in an urban region $(\beta=.13, p<$. $001)$ were most likely to volunteer informally. In conclusion, this study establishes a framework for classifying dimensions of social activity participation that can be used in future research to explore cross-cultural comparisons.

\section{EXPERIENCING CIVIL UNREST: ELDER VOICES ON FERGUSON}

N.L. Morrow-Howell, C. Jackson, S. Herbers, Washington

University in St. Louis, St. Louis, Missouri

Ferguson, Missouri, USA became the center of the nation's attention in August 2014, when an unarmed African American teenager was killed by a Caucasian police officer. Civic unrest continued for weeks. Images and voices in the news were largely of youth; the involvement of older adults was not evident. The aim of Elder Voices on Ferguson was to 
hear how older adults experienced the social unrest and how they thought about improving their community. Ten focus groups were conducted with 73 participants. Focus group recordings were transcribed; and three coders completed a thematic analysis and member checking. Participants averaged 75 years of age, $73 \%$ were female, and $59 \%$ were African American with the remainder White. Eight themes were identified. Issues related to safety were most commonly discussed. Some focus group members participated in the protests but left before nightfall because they felt vulnerable. Concerns about going out after dark remain strong. Some felt intimidated to attend community meetings because of the angry nature of the discussion. There was recognition of ongoing racism and long-standing problems in the community. Participants expressed concern and understanding toward the challenging situations faced by today's youth, including lack of opportunity in education and employment and lack of strong parental and community support. Participants reported a breakdown in intergenerational communications and expressed a desire for more exchange. Findings are being discussed with relevant organizations and public officials to increase the involvement of older adults in on-going community development efforts and to provide opportunities for intergenerational dialogue.

\section{DOES VOLUNTEERING BUFFER HEALTH DURING THE RELOCATION PROCESS? A PROPENSITY SCORE ANALYSIS}

E. Gonzales ${ }^{1,2}$, Y. Wang ${ }^{3}$, H. Shen ${ }^{4}$, T.E. Perry ${ }^{5}$, 1. School of Social Work, Boston University, Boston, Massachusetts, 2. Center for Innovation in Social Work and Health, Boston, Massachusetts, 3. Washington University in St. Louis, St. Louis, Missouri, 4. University of Missouri, St. Louis, St. Louis, Missouri, 5. Wayne State University, Detroit, Michigan

Formal volunteering in later life has been shown to improve health. However, few studies have examined how volunteering can help to buffer health during life-transitions (e.g., relocating from one residence to another). Relocation in later life, at times, is unavoidable. Older adults may move by choice or due to changes in health, widowhood, financial circumstances, etc. Although health outcomes of relocation are mixed, we ask: can volunteering buffer one's health for those who relocate? We utilized 2008 and 2010 Health and Retirement Study and included individuals who were aged $50+$, did not volunteered at baseline, and relocated between 2008 and 2010 ( $\mathrm{N}=835)$. With volunteer engagement as the "treatment," propensity score weighting was performed stepby-step: 1) To estimate the propensity score, the Generalized Boosted Regression (GBR) model was run with a comprehensive set of covariates at the baseline (socio-demographic, health, social network, etc.); 2) Using the propensity score, two types of propensity score weights were calculated: the average treatment effect (ATE), and the treatment effect for the treated (ATT); and 3) To examine the effect of the "treatment," volunteering, on health outcomes in 2010, regression models were performed with ATE and ATT weights. Results suggested engaging in volunteering increased self-report health status; and reduced number of ADL and IADL difficulties. Volunteering did not influence depressive symptoms. Implications for policy and practices intersecting relocation and volunteering will be discussed. Future research should examine the role of specific mechanisms of volunteering (e.g. social engagement, psychological motivations, neighborhood friendliness) and their impacts on health.

\section{ENGAGING AGEING COMMUNITIES AS CO-CREATORS OF SOCIAL SERVICES AND SUPPORT}

S. van der Pas, University of Applied Sciences Leiden, Leiden, Netherlands

As the welfare state retracts, the role of civil society increases. With rising cuts in welfare budgets, there is an increasing need for creative innovations by civil initiatives to cater for welfare and care arrangements. Older adults, social professionals and civil servants take on new roles that require specific capacities/practices of interaction. In this study we apply co-creation as a novel approach to enable older adults to gain ownership over their social and physical environment. Co-creation can be defined as a practice of interactions between older adults, professionals, students, researchers and community stakeholders who jointly define needs and choices as well as design and implement services and support. In a field lab on 'Living Independently for longer', with an elderly community co-operative in the Netherlands, we find that the co-creation approach, fostered empowerment, a more positive image of older adults, and led to the development of age-friendly services and support.

\section{SESSION 5045 (SYMPOSIUM)}

\section{ACEING THE CARE OF OLDER ADULTS IN HOSPITALS THROUGH INNOVATIVE MODELS OF ACUTE CARE FOR ELDERS \\ Chair: S. Sinha, Mt Sinai Hospital, Toronto, Ontario, Canada \\ Co-Chair: N. Foster, Mt Sinai Hospital, Toronto, Ontario, Canada}

At Mount Sinai Hospital, in Toronto Canada, the Acute Care for Elders (ACE) Strategy was conceived as a multicomponent intervention incorporating a series of evidenceinformed but tailored inter-professional interventions (i.e., ISAR Screening, GEM, ACE Units, HELP, House Calls etc.) to improve the care of hospitalized older adults. Starting from fiscal year 2010/11 onwards, a number of evidence informed interventions were gradually implemented each year in a variety of individual patient settings while also looking to address the important issue of transitions especially between hospital and home. The ACE Strategy links these interventions to create a more seamless, integrated inter-professional and team-based delivery-model spanning the continuum of care

Our proposed symposium will explore outcomes related to the implementation of this innovative strategy through four talks: 1) "Establishing the Effectiveness of an Acute Care for Elders (ACE) Strategic Delivery Model" by Dr. Samir Sinha 2) "Measuring the Impact of the GEM Nursing Role in the Emergency Department Setting" by Ms. Nana Asomaning, 3) "Outcomes of a Quality Improvement Intervention to Reduce Unnecessary Urinary Catheter Utilization" by Dr. Richard Norman and 4) "Hospitalization 